\title{
Exploratory Analysis of Biochemical Processes Using Hybrid Modeling Methods
}

\author{
Rimvydas Simutis \\ Institut für Bioverfahrenstechnik und Reaktionstechnik, Universität \\ Halle-Wittenberg, Halle, D-06099, Germany
}

\begin{abstract}
A heuristic process data based procedure has been developed that allows a universal time-efficient exploratory analysis of biochemical processes and predictive information extraction from large data sets. It uses artificial neural networks in combination with mass balance equations to represent unknown process relationships between process variables. An efficient algorithm for training of this hybrid system has been developed. The result of the procedure is a numerical representation of the important process relationships that immediately allows to determine improved set points and/or profiles for the manipulated variables with respect to process performance. For illustration, the procedure is applied for extraction of the important relationships in fed-batch bacterial cultivation process.
\end{abstract}

\section{Introduction}

Since most of the industrial biochemical production plants are equipped with computer based process monitoring units, a large number of measurement data records from production processes is available. Usually, the production process is kept close to its predefined working trajectory. Occasionally, however, deviations from the desired behavior appear due to random fluctuations or small accidents. In this way we also get data from process states around the working trajectory. In this paper, we address the question, how optimally to exploit the information hidden in data records from processes that we do not know precisely enough yet to construct directly a comprehensive process model. In industrial biochemical processes, many variables influence the kinetic quantities such as specific growth and product formation rates, that most often have a predominating influence on the process performance. Usually, it is difficult to disclose the influence of the different state variables on the kinetic expressions using classical statistical methods, since the relevant information must be extracted from measurement data, which is significantly corrupted by measurement noise. As many of the actual process data like biomass, substrate or product concentrations can be measured off-line only, additional difficulties appear due to the long sampling time increments. In this paper, we propose an alternative way. We make use rather of heuristic and intuitive methods, that do not require an extensive knowledge on statistical methods and they can be used semi-automatically directly by the bioprocess engineers that are responsible for running production plants (see Simutis, R., Lübbert, A. 1997). 


\section{Concept of the Exploration Procedure}

\subsection{Formulation of the Problem}

We assume that the dynamics of a biochemical process may be represented in general by the vector differential equation

$$
\frac{d y}{d t}=f\left(y, y_{a}, w, u, t\right)=r\left(y, y_{a}, w, u, t\right) X+\frac{F}{V} \cdot\left(y_{f}-y\right) .
$$

where $y$ is the state vector of the system (e.g. biomass, product concentrations); $y_{a}$ the vector of variables representing the abiotic phase in bioreactor (e.g. temperature, $\mathrm{pH}, \mathrm{pO}_{2}$ ); $w$ is the parameter vector of the model; $u$ is the vector of the variables that can be manipulated; $y_{f}$ is the vector of the concentrations of the different components in the feed added to the bioreactor volume $V$ at a volumetric rate $F ; X$ is the concentration of biomass and $r()$ is the vector of specific rate expressions Such models are of particular interest as long as they can help to optimize the process. In typical biochemical process optimization tasks the performance index or objective function is indirectly determined by the specific growth rate and the specific product formation rate. Hence, the essential practical problem is to determine the specific rate expressions $r()$, and, in particular, to determine their functional relationships with the state variables $y$ and the variables $y_{a}$ characterizing the abiotic phase of the culture. Usually, the response of the cells to the changes in the culture medium is not known exactly, even in ideal bioreactors. The effective relationships depend on the reactor transport properties that are scale-dependent. For a given reactor, however, it is often sufficient in practice to approximate these expressions around the actual working points by means of simple black box relationships. An often used black-box approach is the polynomial representation of $r()$. The classical approach of identification of the rates $r()$ from measurements is to smooth the data by means of splines [1]. The smoothed data have been used to determine a first estimation of the reaction rates.Then least square techniques can then be used to determine the parameters (coefficients) of the polynomials. This procedure is referred to as the differential approximation method [2]. A disadvantage of this technique is that the estimation errors of the specific rates and the other parameters may become considerable, when the relevant sampled data are available with large time increments only. The accuracy sensitively depends on the degree of smoothing which, consequently, must be performed carefully in order to obtain the desired estimation quality. In our experience, polynomials are of much restricted flexibility in approximation of the true kinetic expressions. Recently, Royston and Altman [8] have developed a family of 'fractional polynomial' models, which seems to have more flexibility than conventional polynomials to fit functional relationships and which eventually will create new opportunities for the modeling.

In this paper, we focus our attention on artificial neural networks (ANN) representations of the nonlinear relationships between the specific rates and the state variables as well as the variables that characterize the abiotic phase of the culture. In order to show the advantages of these particular representations as 
compared with the conventional approach, we also prepared polynomial representations of these relationships.

\subsection{Hybrid Modeling Approach}

The proposed procedure may be used to determine the influence of the different process variables on the specific reaction rates like the specific growth rate and specific product formation rate. It was worked out for practical applications where a large number of measurement data records is available. This condition is fulfilled at almost all industrial production processes. The specific rate expressions $r()$ are represented using artificial neural network, where the ANN inputs are different process variables. The resulting $r()$ are immediately used as input quantities in the set of mass balance equations (see Psichogios, D. Ungar, L. 1992, Schubert, J., et. al. 1994).

In order to train the neural network part of the combined process model, pairs of input/output data vectors, as measured at the real plant are to be used. The training requires that the ANN weights $w$ are determined in such a way that the sum of the squared deviations, $J$, between the output data $y_{i}$ predicted by the combined model and the corresponding process data $y_{i e x p}$ becomes minimal:

$$
J=0.5 \sum\left(y_{i}-y_{i e x p}\right)^{2}=>\min .
$$

The usual way to minimize $J$ is to use gradient methods for adapting of ANN weights $[4,13]$ :

$$
w_{n+1}=w_{n}-g \cdot \partial J / \partial w_{n},
$$

where $g$ is a gain factor, determined by experience. By forming the derivative of $J$ with respect to $w_{n}$, we find

$$
\partial J / \partial w_{n}=\sum\left(y_{i}-y_{i e x p}\right) \cdot \partial y_{i} / \partial w_{n},
$$

hence, for ANN tuning, it is necessary to determine the derivatives $\partial y_{i} / \partial w_{n}$. In the combined system, however, system outputs $y$ are different from the outputs of the neural network, therefore a training of ANN with application of conventional backpropagation technique is impossible. A promising alternative is the sensitivity approach based on a concept developed in system engineering (see Frank, P. 1978). In order to calculate gradients $\partial y_{i} / \partial w_{n}$ it is of advantage to differentiate equation (1) with respect to weights $w_{n}$, leading to

$$
\frac{d}{d t} \cdot \frac{\partial y_{i}}{\partial w_{n}}=\frac{\partial f}{\partial y} \cdot \frac{\partial y_{i}}{\partial w_{n}}+\frac{\partial f}{\partial w_{n}} .
$$

Eq. (5) is a differential equation for the unknown gradients $\partial y_{i} / \partial w_{n}$ required in the ANN training algorithm. The initial condition at time $t=0$, necessary to solve the equation (5), is known to be

$$
\frac{\partial y_{t=0}}{\partial w_{n}}=0 .
$$


When the specific rate expressions $r()$ are represented by feedforward ANN, the matrix $\partial f / \partial w_{n}=\partial r / \partial w_{n}$ can be readily computed by using the standard backpropagation method. This is the fact that makes ANN so attractive for mapping of specific reaction rates $r()$. In this hybrid modeling structure, the ANN is trained directly on the original concentration data (off-line measurements). It is not necessary to calculate derivatives of the smoothed concentration values as in the differential approximation technique. The training procedure for the combined system of the ANN and the mass balance equations can be regarded as an optimization procedure that minimizes the deviations of the estimated values of the process variables from the measured ones. The general procedure of solving the ANN training problem is:

1. Set up of the global balance equations.

2. Choosing the structure and start weights of ANN.

3. Establishing the sensitivity equations.

4. Integrating the sensitivity equations and determining $\partial y / \partial w_{n}$.

5. Making use of $\partial y / \partial w_{n}$ in the same way as with classical ANN.

In order to reduce training time for discussed application, the training was carried out with a combination of the classical backpropagation technique and a conjugate gradient optimization method. In order to achieve better process representation, the cross validation technique during the ANN training procedure was used (see Pollard, J., et al. 1992). The concept of cross validation is that after training using a given sample of data ('training set') the quality of the process representation is evaluated using a different data set ('test set'). The root mean square error (RMSE) between predicted and measured outputs in the 'test set' is referred to as the 'test error'. The best process representation is defined as the one which minimizes the 'test error'.

\subsection{Basic Exploration Procedure}

The proposed algorithm can be divided into two major parts. The first part of the algorithm (referred to as method 1) can be solely used in the case where it can be assumed that the process variables considered do not show cooperative effects, the entire algorithm (called method 2) can be used for the general case. The algorithm is started with a first guess about the basic process variables that must be considered in the numerical process representation. The choice of inputs for ANN models is crucial, because the addition of input variables that are essentially irrelevant will always degrade the process representation [5]. In most biotechnological processes the substrate and biomass concentration (batch processes) or specific substrate feed rate (fed-batch processes) are good examples of the basic variables. Once the basic input variables are chosen, they are used as input variables for the first 'basic ANN'. Then this is trained on the 'training set'. The used training procedure is already discussed sensitivity equation-based conjugate gradient optimization method. The weights of this 'basic ANN' are stored since they will repeatedly be used in followed exploration. In order to improve the basic process representation, it is necessary to find additional process 
variables that, if considered, will improve the accuracy of the process representation. The structure of proposed exploration procedure is sketched in Figure 1. The 'basic cycle' of the exploration procedure is the following: In every run

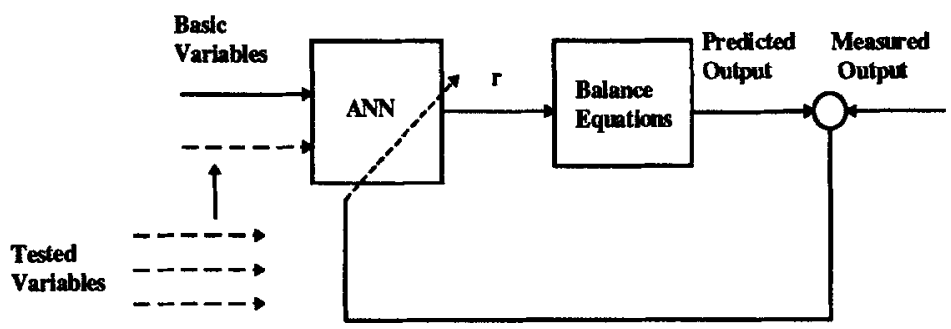

Fig. 1. Structure of the exploration procedure that is based upon a hybrid modeling methods

of the cycle it is started with the trained 'basic ANN'. Then this 'basic ANN' is extended by a single input variable from the set of additional variables. In the beginning of the 'basic cycle' the weights of the nodes in the hidden layer connected with the new input variable are set close to zero, in order to start with a negligible influence on the network by this additional variable. Then, the extended network is trained on the corresponding data from the 'training set'. The improvement of the representation by this training is measured by means of the corresponding development of the 'test error'. After running the 'basic cycle' with all the additional process quantities one after another, always starting with the same 'basic ANN', the variable with the biggest 'test error' reduction is found. This variable can be considered to be the next candidate to extend the model, if this action leads to significant advantage. As a rule of thumb, the improvement in the 'test error' may be considered to be significant if the reduction in the 'test error' is larger than 5\%. All variables that do not reach such a big influence are considered as insignificant to the objectives of the model. Hence, they are not considered further, since they only confuse the artificial network and decrease the model's forecasting ability.

At the end of each run of the 'basic cycle', some information about the influence of the additional variables under consideration can be obtained. By means of a systematic variation of the values of the additional variable at the trained extended network, plots of specific rates against this variable can be generated. Such plots can be used to generate the first guess of a set point/profile for a process controller. Else, the shape of the curve can be used to extract some valuable information about the sensitivity of the variable under consideration with respect to the specific rates. 


\subsection{Extension of the Basic Exploration Procedure}

The basic exploration procedure discussed above can be expanded by increasing the number of input variables of the 'basic ANN'. It is straightforward to add the variable that, during the application of the 'basic cycle', turned out to provide the biggest reduction of the 'test error'. In this way we get a new extended 'basic ANN' representing the process kinetic relationships. Then, with this updated ANN the 'basic cycles' procedure is repeated and again, the variable that leads to the smallest 'test error' is used to extend the 'basic ANN'. The threshold value of the change in 'test error' for the influence of the variable to be considered as significant is the same as in the first part of the algorithm. This extension cycle is repeated until no more free additional variables are available. The procedure can be viewed at as a method of ranging or classifying the additional variables. A similar procedure is used in image analysis, where it is known as ANN growing procedure (see Haykin, S. 1994). The approach used here is adjusted to dynamical systems. The application of this extended procedure is necessary in all the cases where a cooperative influence of more than a single variable on the specific reaction rate is to be expected. However, the extended exploration method does not only provide information about the variables that may influence the key quantities of the bioprocess (here, the specific reaction rates), it also provides a data driven gray-box model, which can be used to determine the optimal set-points or profiles for the control variables.

\section{Application of the Exploration Procedure}

Generally, we use fully connected single-hidden layer feedforward networks with a hyperbolic tangent activation function [4] as the basis network type in the proposed hybrid process representation structure. The number of nodes in the hidden layer is typically from 10 to 15 . This number proved to be sufficient to approximate the kinetic realationships typical to biotechnology. In the beginning, of the training procedures the ANN is initialized with small randomly chosen weights. The training was based on the sensitivity equation approach discussed above. For the optimization, the conjugate gradient scheme and the E06DFG routine of the NAG-library have been used. The algorithms are processed under the software package HYBNET [10] on DEC Alpha. It proved to be of advantage with respect to learning time and efficiency of exploration procedure to work with transformed data, i.e., data with zero mean and unit variance. The corresponding averages and variances must be estimated for all the process variables. Needless to say that before starting with this exploration procedure, a careful data preprocessing is required, in which all systematic errors and outliners are eliminated. This will reduce the computational load and avoid confusing the networks with wrong data. Since the training procedure proposed for the hybrid modeling approach is based on gradient optimization methods, it is strongly recommended to repeatedly perform the exploration analysis with different start vectors for the ANN-weights. Finally, the network that led to the minimal 'test error' should be taken. Here we present the application of the proposed exploration procedure for 
the identification of relationships between the specific growth rate $\mathrm{r}=\mu$ and the additional bioprocess variables in fed-batch bacterial cultivation process. The scheme of the task is sketched in Figure 2. The different bioprocess variables are

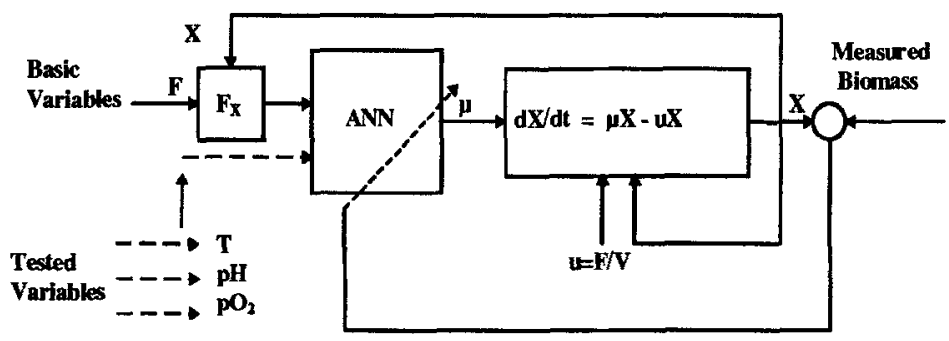

Fig. 2. Exploration procedure for fed-batch bacterial cultivation process

used as ANN-inputs for the specific growth rate representation and output of the net is connected with the balance equation block. The data sets were used to train the ANN-based process representations as well as to identify the coefficients of the polynomials in the conventional differential approximation aproach. Then the process representations obtained from this data were compared with the true process relationships (process model). The quality of the process representation is evaluated using the root mean square error (RMSE) between both relationships and is referred as the 'modeling error' in the following text. In order to provide an objective base for comparisons, the proposed algorithms as well as the conventional polynomial approach are applied to simulated data generated with a given classical process model. The example chosen is a model of a fed- batch bacterial cultivation process in which the specific growth rate $\mu$ is influenced by 4 variables: specific glucose feed rate, temperature, $\mathrm{pH}$ and pO2. Additionally, a variable that does not have any influence on the growth rate is added to the data sets in order to make sure that the procedure is also capable of recognizing variables that do not make any influence on the growth rate. The off-line measured biomass values are disturbed by equally distributed random noise. Data with different relative noise levels between 1 and $10 \%$ were taken in order to estimate the efficiency of the proposed methods at different measurement-noise levels. The plant imitation model and the data generation procedure are described in details by Simutis and Lübbert [10]. A total of 70 fermentation runs were simulated. Each data set comprises signals of the glucose feed rate, culture volume,temperature, $\mathrm{pH}, \mathrm{pO}_{2}$, noise and off-line measurement data of the biomass. Same examples of simulated data records generated with a bacterial growth model are depicted in Figure 3. In accordance to the rule of thumb for cross validation procedure [4], 50 of the simulated data sets are taken for the 'training set', the rest for cross validation of the hybrid model, i.e. for the 'test set'. 

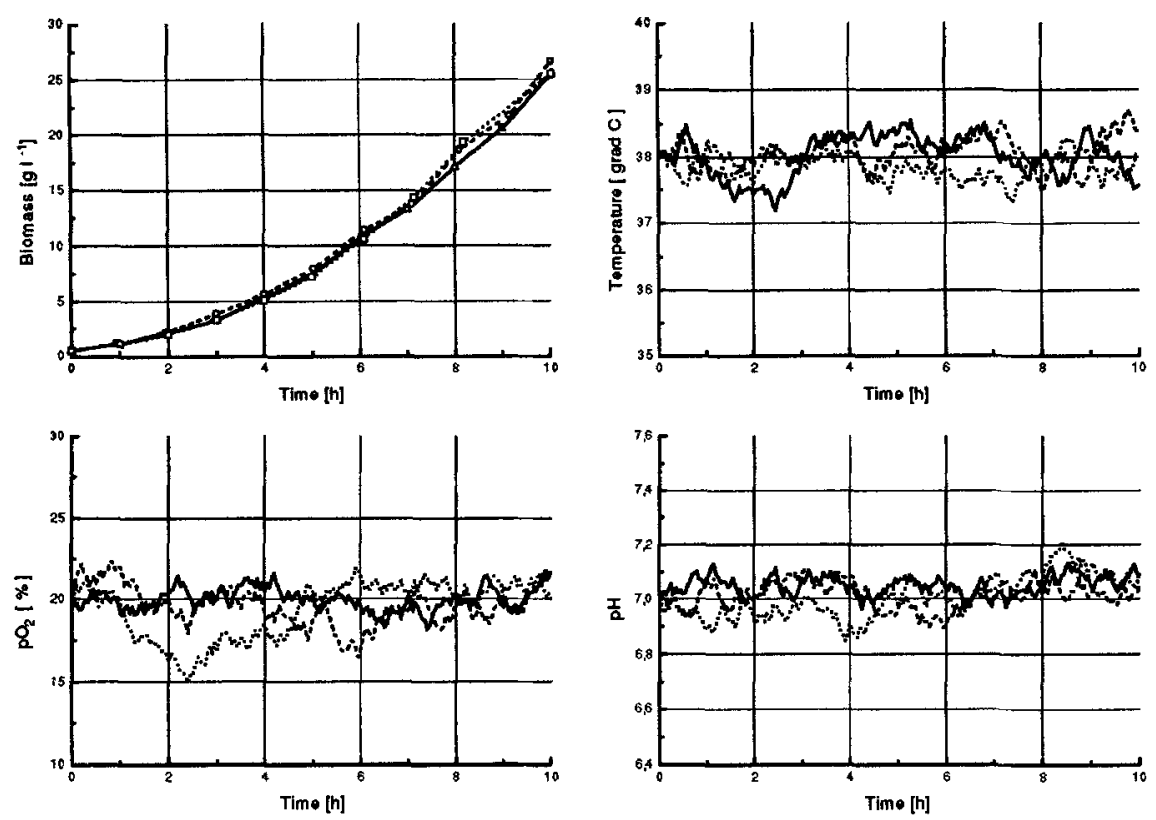

Fig. 3. Examples of simulated data records generated with a bacterial growth model

\subsection{Results Obtained with the Basic Procedure}

The elementary exploration procedure proposed, here referred to as method 1 , was able to provide the correct form of the dependency between the variables considered and the specific growth rate for all noise levels investigated. Some results of the analysis are depicted in Figure 4 (case with $5 \%$ relative noise in biomass measurements). The simple method was able to classify the noise signal correctly as insignificant input signal in all cases. Also, the root-meansquare error between the true relationship and the relationship recognized by the exploration procedure was lower as the comparable one obtained with the conventional polynomial approach in all investigated cases. However, this simple version of the exploration procedure does not provide sufficiently accurate results when the measurement noise is greater than $5 \%$ in biomass measurement. Nevertheless the technique can be used as a first approach to a (coarse) process analysis.

\subsection{Results Obtained with the Extended Procedure}

In Table 1 an example of the stepwise extension of the 'basic ANN' and the evolution of the 'test error' is shown. As the basic variable, the specific glucose feed rate is chosen. Then, one after another, the influence of temperature $\mathrm{T}$, dissolved oxygen tension $\mathrm{pO}_{2}, \mathrm{pH}$ and some insignificant noise signal were tested. 

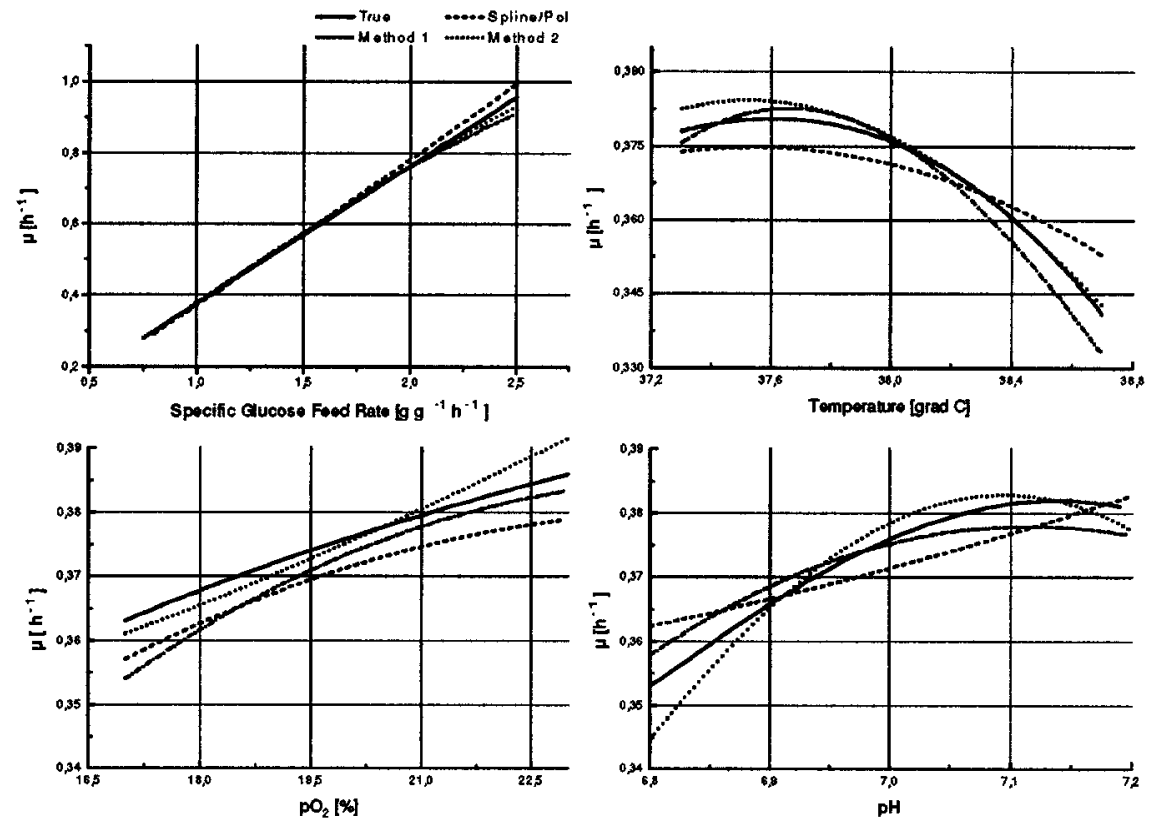

Fig. 4. Relationships between the specific growth rate and the considered variables as estimated by different methods discussed in the text

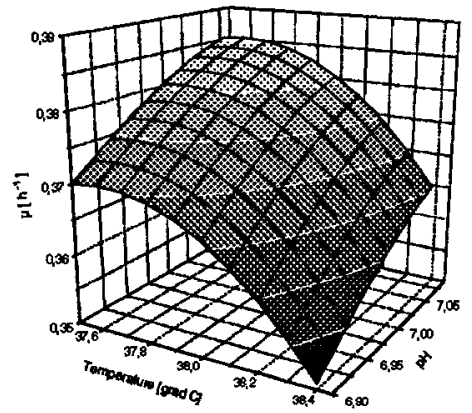

a)

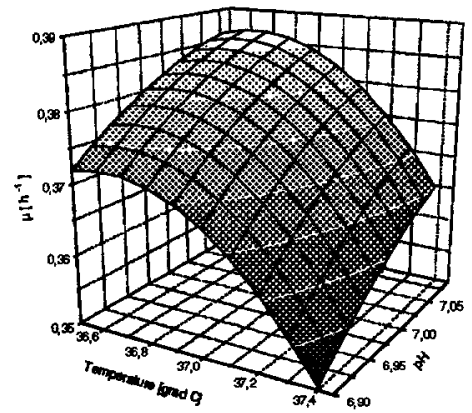

b)

Fig. 5. Example of the true specific growth rate relationship (a) and the relationship estimated with the Method 2 (b) as a function of the temperature $\mathrm{T}$ and the $\mathrm{pH}$-value 
Table 1. Protocol of the stepwise extension of the basic ANN by the different additional process variables as explained in the text. The results are shown for the case of $5 \%$ noise in the off- line biomass measurements

\begin{tabular}{|c|c|c|c|}
\hline Exploration Step & Basic Variables & Tested Variables & RMSE in Test Set \\
\hline 1 & $F_{X}$ & $\begin{array}{c}- \\
\mathrm{T} \\
\mathrm{pO}_{2} \\
\mathrm{pH} \\
\text { Noise }\end{array}$ & $\begin{array}{l}0.382 \\
0.315 \\
0.357 \\
0.343 \\
0.380\end{array}$ \\
\hline 2 & $\underset{\mathrm{T}}{\mathrm{F}_{\boldsymbol{X}}}$ & $\begin{array}{c}- \\
\overline{\mathrm{pO}_{2}} \\
\mathrm{pH} \\
\text { Noise }\end{array}$ & $\begin{array}{l}0.315 \\
0.280 \\
0.292 \\
0.312 \\
\end{array}$ \\
\hline 3 & $\begin{array}{c}\mathrm{F}_{X} \\
\mathrm{~T} \\
\mathrm{pO}_{2}\end{array}$ & $\begin{array}{c}- \\
- \\
- \\
\text { pH } \\
\text { Noise }\end{array}$ & $\begin{array}{l}0.280 \\
0.240 \\
0.277 \\
\end{array}$ \\
\hline 4 & $\begin{array}{c}\mathrm{F}_{\boldsymbol{X}} \\
\mathrm{T} \\
\mathrm{pO}_{2} \\
\mathrm{pH}\end{array}$ & $\begin{array}{c}- \\
- \\
- \\
- \\
\text { Noise }\end{array}$ & 0.236 (non-significant) \\
\hline
\end{tabular}

est 'test error'. Hence, the temperature was introduced into the updated 'basic ANN'. Once again, the 'basic cycles' were applied with the rest of the additional variables, here with $\mathrm{pO} 2, \mathrm{pH}$ and the noise. In this round, the $\mathrm{pO} 2$ turned out to lead to the lowest 'test error'. Thus, it was put into the next update of the 'basic ANN' and the procedure was repeated in the same way until no further additional variable is left. With this procedure improved kinetic relationships can be obtained, as shown in Figure 4 . In this case the variable influence curves were obtained by keeping all, but the single additional variable considered, constant. The latter was systematically varied around it's assumed working point. In the same way, subsurfaces can be constructed by varying two of the additional variables. Figure 5 depicts an example of such a surface of $\mu$ on the $\left(\mathrm{T}, \mathrm{pO}_{2}\right)$-plane, both for the true and for the relationship evaluated with method 2 (for the case $3 \%$ noise in biomass off-line measurement). As compared to the classical spline approach, the method proposed here, leads to a significantly higher quality. The most important obtained result is that the ANN-based recognition of the relationships is sufficient with respect to the form of the curves and the quantitative In this round of 'basic cycles' the temperature turned out to lead to the low- 
agreement as long as the noise level of the data is up to about $10 \%$. At higher noise levels, the influence of some of the variables on specific growth rate can still be recognized, however, the results do not match quantitatively. Applications of the extended exploration procedure essentially result in process models with a substantial representation capacity. It needs considerable computation time, more than the classical procedure or the simple exploration technique, however, its advantage is that the final version of the resulting representations of the kinetic expressions is by our experience of sufficient quality that these expressions can immediately be used in process optimization studies.

\section{Discussion}

In a big volume production process, even small improvements in the productivity can be attractive for the company. Here we discuss a new technique, which allows a data based extraction of important realationships in the industrial biochemical processes. It makes use of the artificial neural networks in combination with mass balance equations to represent the process dynamics. The applicability of the method is restricted to the relatively narrow area of the state-space from where measurement data already exists. Its big advantage, however, is that such hybrid process representations can be created quickly and semi-automatically, and can easily be adapted to changing conditions of the process.

\section{References}

1. Eubank, R. Spline Smoothing and non-parametric regression. Marcel Dekker, New York, 1988

2. Eykhoff, P. System identification: Parameter and state estimation. John Wiley Sons, New York, 1974

3. Frank P, M. Introduction to System Sensitivity Theory. Academic Press, New York, 1978

4. Haykin, S. Neural networks. A comprehensive foundation. Macmillan College Publishing Company, New York, 1994

5. Kell, D.B., Sonnleitner, B.: GMB - Good modelling practice: an essential component of good manufacturing practice. Trends Biotechnol.13 (1995) 481-492

6. Pollard, J.F., Broussard, M.R., Garrison, D.B., San, K.Y.: Process identification using neural networks. Comp.Chem.Eng.16 (1992) 253-270

7. Psichogios, D.C., Ungar, L.H.: A hybrid neural network - first principles approach to process modeling. AIChE J. 38(1992) 1499-1511

8. Royston, P., Altman D., G.: Using fractional polynomials to model curved regression relationships. Stata Technical Bulletin. 21 (1994) 11-23

9. Schubert, J., Simutis, R., Dors, M., Havlik, I., Lübbert, A.: Bioprocess optimization and control: Application of hybrid modeling. J. Biotechn. 35 (1994) 51-68

10. Simutis, R., Lübbert, A.: Exploratory analysis of bioprocesses. Biotechnol.Prog. (to appear) 